\title{
The Serum Assay of Soluble CD44 Standard, CD44 Variant 5, and CD 44 Variant 6 in Patients with Gastric Cancer
}

\author{
Chang Hak Yoo, M.D. ${ }^{1}$ and Sung Hoon Noh, M.D. ${ }^{2}$ \\ Department of Surgery, ${ }^{1}$ Kangbuk Samsung Hospital, Sungkyunkwan University School of Medicine, ${ }^{2}$ Yonsei University \\ College of Medicine, Seoul, Korea
}

Purpose: Alternative splicing of CD44 and aberrant levels of soluble CD44 (sCD44) protein in the serum of cancer patients has been correlated to tumor progression and metastasis. The purpose of this study was to evaluate the concentrations, and the prognostic potential of sCD44s, sCD44v5 and sCD44v6, in patients with gastric cancer.

Materials and Methods: The serum levels of SCD44s, sCD44v5 and sCD44v6 were determined quantitatively using an enzyme-linked immunosorbent assay. Serum samples were obtained from 116 patients with gastric cancer, both before and after surgery, and from 30 healthy controls.

Results: The serum SCD44v6 levels were significantly higher in patients with gastric cancer than in the healthy controls, whereas those of SCD44s and SCD44v5 were no different. The surgical resection of the tumor resulted in a significant reduction in all the SCD44 proteins, whereas if a surgical resection was not performed the concen-

\section{INTRODUCTION}

In malignant diseases, characteristic alterations in the expression of numerous cell adhesion molecules have been detected. One of these interesting proteins is CD44 (1). CD44 was first described by Jalkanen et al. (2) as a lymphocyte-homing receptor, regulating the entry of circulating lymphocytes into the lymphatic tissue by its attachment to endothelial venules. However, this adhesion molecule is not only present on lymphocytes, but is ubiquitously expressed on cells of mesodermal and hematopoietic origins (3). CD44 acts as a principal receptor for hyaluronate, and is involved in cell-cell or cell-matrix interactions (4). The CD44 gene consists of 20 exons, ten of which are

Correspondence: Chang Hak Yoo, Department of Surgery, Kangbuk Samsung Hospital, Sungkyunkwan University School of Medicine, 108 Pyeong-dong, Jongno-gu, Seoul 110-102, Korea. (Tel) 02-2001-2460, (Fax) 02-2001-2131, (E-mail) chyoo63@ naver.com

Received November 25, 2002, Accepted February 10, 2003 trations of the SCD44v5 and SCD44v6 were not reduced prior to surgery. The serum SCD44v6 levels correlated with the venous or lymphatic invasion of the tumor and lymph node metastasis. In addition, a high preoperative serum sCD44v6 level was significantly associated with poor prognosis in patients with gastric cancer.

Conclusion: The preoperative serum level of SCD44v6 in patients with gastric cancer was significantly higher than that in the healthy controls, and correlated with the venous or lymphatic invasion of the tumor and lymph node metastasis. In addition, a high preoperative serum sCD44v6 level was significantly associated with poor prognosis in patients with gastric cancer. These results suggest that an elevation of the serum SCD44v6 level might be used as a new predictor of tumor invasiveness, and poor prognosis, in patients with gastric cancer. (Cancer Research and Treatment 2003;35:3-8)

Key Words: Stomach neoplasm, CD44, Prognosis constitutively expressed in the standard CD44 form (CD44s). At least 20 variants of $\mathrm{CD} 44$, the products of the alternative messenger RNA splicing of the remaining ten exons (v1-v10) encoding the proximal portion of the extracellular domain of the membrane, have been isolated and sequenced (5).

Recently, the expression of CD44s, as well as those of certain splicing variants, have been found in various types of human malignancies, and are considered to implicate tumor progression and metastasis (6). In gastric cancer, the expression of CD44 also correlates with a more aggressive tumor type, and the differential expression of certain splice variants has been shown for intestinal- and diffuse-types of gastric carcinomas (7 13).

In contrast to the evaluation of the CD44 expression in tumor tissue, the measurement of soluble CD44 molecules in easily available body fluids could be a simple and useful tool in cancer diagnostics, and for monitoring the efficiency of treatment. The soluble form of CD44 (sCD44) is present in the serum, and can be detected by enzyme-linked immunosorbent assay (ELISA) or Western blot analysis. The level of sCD44 in serum has been observed to correlate with tumor burden and metastasis in patients with gastric and colorectal cancer (14). Furthermore, Harn et al. (15) reported that the concentrations 
of sCD44v5 and sCD44v6 were significantly elevated in patients with advanced gastric cancer.

In this study, we examined the concentrations of sCD44s, and the isoforms containing sCD44v5 and sCD44v6, in the sera of patients with gastric cancer. We then looked at the implications of the results, with respect to the clinicopathological features and prognosis of our patients, to evaluate their potential usefulness as markers in patients with gastric cancer.

\section{MATERIALS AND METHODS}

\section{1) Patients and specimens}

Serum samples were obtained from 116 patients with primary gastric carcinoma (79 male, 37 female) who were admitted to the Department of Surgery, Yonsei University College of Medicine, between July 1997 and December 1997. The mean age was 56 years (range 26 78 years). In 96 patients (82.7\%), a tumor resection was performed (total gastrectomy in 37 , and subtotal gastrectomy in 59); and in the other 20 (17.3\%), an explorative laparotomy or by-pass procedures were performed. All blood samples were taken by intravenous puncture on the morning before surgery and postoperative day 7 , and were stored at $-70^{\circ} \mathrm{C}$ until assayed. Control sera were obtained from 30 age- and sex-matched healthy volunteers (11 female, 19 male; mean age, 53 years; range $32 \sim 71$ years).

The clinicopathological findings and staging of the cancers were determined according to the rules of the Korean Classification of Gastric Cancer (16), and the Union Internationale Contre le Cancer TNM Classification (17). All patients were followed closely until December 2001; the median length of follow-up was 38 (range 1 62) months.

\section{2) ELISA}

The serum levels of soluble CD44s, CD44v5 and CD44v6 were measured using a commercially available ELISA kit (Bender MedSystems, Vienna, Austria). According to the data given by the manufacturer, the respective assays specifically recognize the corresponding sCD44 molecules. The antibodies and their specificities have been published. There was no detectable cross-reactivity with circulating factors of the immune system (e.g. TNF- $a$, TNF- $\beta$ ) or CD44 polypeptides lacking the protein sequence encoded by the corresponding exons. Briefly, the anti-CD44s, -v5 or v6 monoclonal antibodies were adsorbed onto microwells of 96-well microtiter plates. The CD44 present in samples or standards were allowed to bind to the antibodies adsorbed onto the microwells; a horseradish peroxidase (HRP)-conjugated monoclonal anti-CD44 antibody was added, and allowed to bind to the CD44 captured by the first antibody. The unbound enzyme-conjugated antiCD44 was removed by washing, and an HRP substrate solution was added to the wells. A colored product formed in proportion to the amount of CD44 present in samples. The reaction was terminated by the addition of acid, and the absorbance measured at $450 \mathrm{~nm}$. A standard curve was prepared from six standard dilutions, which allowed determination of the CD44 concentrations in the samples. The assays were repeated twice, with similar results.

\section{3) Statistical analysis}

All results are expressed as the mean \pm standard deviation. The significance of differences among the means was determined by the Student's t-test and a one-way analysis of variance. Survival curves were drawn using the Kaplan-Meier method, and the comparisons of the survival distribution were made using the log-rank test. The prognostic relevance was verified by applying a multivariate Cox regression analysis. $\mathrm{p}$ values $<0.05$ were considered statistically significant. All the statistical analyses were carried out using SPSS 10.0 for Windows (SPSS Inc., Chicago, IL).

\section{RESULTS}

Different results for the preoperative sCD44s, sCD44v5 and sCD44v6 serum levels, between gastric cancer patients and the healthy controls were obtained. Whereas, the sCD44v6 serum levels were significantly higher in patients with gastric cancer than in the healthy controls $(\mathrm{p}=0.004)$, but the $\mathrm{sCD} 44 \mathrm{~s}$ and sCD44v5 serum levels were no different between the groups (Table 1).

The correlations between the serum sCD44s, sCD44v5 and sCD44v6 concentrations, and the different clinicopathological variables, are shown in Table 2. Other clinicopathological variables, such as patient age, sex and tumor size and location were found not to be associated with the sCD44s, sCD44v5, and sCD44v6 serum levels (data not shown). With the sCD44s, the histological tumor type had a significant difference in patients with gastric cancer $(p=0.001)$. The serum sCD44v5 levels showed a significant correlation with the histological tumor type $(p=0.021)$ and venous invasion $(p=0.006)$. The serum $\mathrm{sCD} 44 \mathrm{v} 6$ levels in patients with venous $(\mathrm{p}=0.043)$, or lymphatic $(p=0.038)$, invasions were significantly higher than those with no such invasion. We also found that the serum sCD44v6 levels in patients with lymph node metastasis were significantly higher than those without $(\mathrm{p}=0.043)$.

The serum concentrations of sCD44s, sCD44v5 and sCD44v6, before and after surgery, are shown in Table 3. All the CD44 serum levels, measured on 7 days postoperatively, were significantly decreased. We compared the pre- and postoperative CD44 serum levels in those patients who underwent curative, or palliative, resection of gastric cancer with those who did not.

Table 1. Preoperative serum concentrations of sCD $44 \mathrm{~s}, \mathrm{sCD} 44 \mathrm{v} 5$, and $\mathrm{sCD} 44 \mathrm{v} 6$ in healthy controls and patients with gastric cancer

\begin{tabular}{lccc} 
& & Average concentration $($ mean $\pm \mathrm{SD}, \mathrm{ng} / \mathrm{ml})$ & \\
\cline { 2 - 3 } $\mathrm{sCD} 44$ & $\begin{array}{c}\text { Control } \\
(\mathrm{n}=30)\end{array}$ & $\begin{array}{c}\text { Gastric cancer } \\
(\mathrm{n}=116)\end{array}$ & \\
& & & \\
\hline SCD44alue & & \\
sCD44v5 & $353.8 \pm 60.1$ & $400.9 \pm 135.4$ & 0.066 \\
sCD44v6 & $28.5 \pm 18.1$ & $24.7 \pm 20.6$ & 0.362 \\
& $194.8 \pm 45.7$ & $243.0 \pm 86.6$ & 0.004
\end{tabular}


Chang Hak Yoo, et al : Soluble CD44 Isoforms in Gastric Cancer 5

Table 2. Serum concentrations of sCD44s, sCD44v5, and sCD44v6 in relation to clinicopathologic features in patients with gastric cancer

\begin{tabular}{|c|c|c|c|c|}
\hline \multirow{2}{*}{ Variables } & \multirow{2}{*}{$\begin{array}{l}\text { No. of } \\
\text { patients }\end{array}$} & sCD44s & sCD44v5 & sCD44v6 \\
\hline & & \multicolumn{3}{|c|}{$(\mathrm{mean} \pm \mathrm{SD}, \mathrm{ng} / \mathrm{ml})$} \\
\hline \multicolumn{5}{|l|}{ Gross type } \\
\hline Superficial or localized & 44 & $423.2 \pm 128.9$ & $21.6 \pm 18.2$ & $232.2 \pm 49.8$ \\
\hline Infiltrative or diffuse & 72 & $387.2 \pm 138.4$ & $26.7 \pm 21.9$ & $249.6 \pm 92.3$ \\
\hline \multicolumn{5}{|l|}{ Histologic type } \\
\hline Differentiated & 39 & $337.8 \pm 101.3^{*}$ & $18.5 \pm 12.7$ & $231.6 \pm 55.2$ \\
\hline Undifferentiated & 77 & $432.8 \pm 139.8$ & $27.9 \pm 23.1$ & $248.8 \pm 88.6$ \\
\hline \multicolumn{5}{|l|}{ Lauren type } \\
\hline Intestinal & 50 & $415.7 \pm 122.8$ & $22.1 \pm 17.6$ & $232.1 \pm 47.0$ \\
\hline Diffuse & 66 & $389.6 \pm 144.1$ & $26.7 \pm 22.6$ & $251.3 \pm 96.2$ \\
\hline \multicolumn{5}{|l|}{ Venous invasion } \\
\hline Negative & 74 & $392.5 \pm 128.2$ & $22.1 \pm 15.1^{*}$ & $233.3 \pm 56.8^{\dagger}$ \\
\hline Positive & 22 & $417.6 \pm 147.9$ & $34.0 \pm 23.3$ & $263.9 \pm 63.3$ \\
\hline \multicolumn{5}{|l|}{ Lymphatic invasion } \\
\hline Negative & 56 & $404.7 \pm 131.7$ & $22.2 \pm 13.8$ & $232.2 \pm 54.7^{\dagger}$ \\
\hline Positive & 40 & $389.2 \pm 135.0$ & $28.9 \pm 21.9$ & $259.3 \pm 64.6$ \\
\hline \multicolumn{5}{|l|}{ Serosal invasion } \\
\hline Negative & 38 & $412.5 \pm 139.6$ & $22.5 \pm 13.0$ & $239.8 \pm 57.4$ \\
\hline Positive & 78 & $395.2 \pm 133.8$ & $25.8 \pm 23.5$ & $249.6 \pm 88.1$ \\
\hline \multicolumn{5}{|l|}{ Lymph node metastasis } \\
\hline Negative & 40 & $393.1 \pm 113.3$ & $22.1 \pm 11.8$ & $230.6 \pm 60.7^{\dagger}$ \\
\hline Positive & 76 & $404.9 \pm 146.3$ & $26.1 \pm 24.0$ & $256.8 \pm 86.1$ \\
\hline \multicolumn{5}{|l|}{ Distant metastasis } \\
\hline Negative & 86 & $408.3 \pm 135.3$ & $25.1 \pm 17.8$ & $241.1 \pm 61.4$ \\
\hline Positive & 30 & $379.5 \pm 135.8$ & $23.9 \pm 27.5$ & $248.6 \pm 117.2$ \\
\hline \multicolumn{5}{|l|}{ Stage } \\
\hline $\mathrm{I} / \mathrm{II}$ & 46 & $409.9 \pm 135.5$ & $21.7 \pm 12.7$ & $234.7 \pm 60.6$ \\
\hline III/IV & 70 & $394.9 \pm 136.1$ & $26.7 \pm 24.4$ & $248.5 \pm 89.2$ \\
\hline
\end{tabular}

${ }^{*} \mathrm{p}<0.01,{ }^{\dagger} \mathrm{p}<0.05$

Table 3. Serum concentrations of sCD44s, sCD44v5, and sCD44v6 before and after surgery

\begin{tabular}{cccc} 
& Before surgery & After surgery & P-value \\
\hline $\begin{array}{c}\text { All patients } \\
\text { (n=116) }\end{array}$ & & & \\
sCD44s & $400.9 \pm 135.4$ & $165.1 \pm 77.5$ & $<0.001$ \\
sCD44v5 & $24.7 \pm 20.6$ & $15.6 \pm 19.1$ & $<0.001$ \\
SCD44v6 & $243.0 \pm 79.1$ & $192.8 \pm 82.9$ & $<0.001$ \\
Resection group & & & \\
(n=96) & & & \\
SCD44s & $398.3 \pm 132.6$ & $147.2 \pm 55.4$ & $<0.001$ \\
sCD44v5 & $24.9 \pm 17.8$ & $13.8 \pm 12.1$ & $<0.001$ \\
sCD44v6 & $242.6 \pm 60.0$ & $182.1 \pm 55.9$ & $<0.001$ \\
Non-resection group & & & \\
(n=20) & & & \\
sCD44s & $413.5 \pm 151.4$ & $250.7 \pm 107.9$ & $<0.001$ \\
sCD44v5 & $23.6 \pm 21.4$ & $24.5 \pm 19.1$ & 0.557 \\
sCD44v6 & $244.8 \pm 141.0$ & $244.4 \pm 150.1$ & 0.939 \\
\hline
\end{tabular}

In the resection group, all the CD44 serum levels were significantly decreased after the operation, whereas the serum sCD44v5 and sCD55v6 levels in the non-resection group were not.

Fig. 1 shows the Kaplan-Meier survival curves of the 116 patients, divided into two groups, according to the concentrations of sCD44s, sCD44v5 and sCD44v6. When the mean serum levels were used as a cut-off value, the 5-year survival rate of the patients in the high level sCD44v6 (40.4\%) group was significantly lower than that of the patients in the low level group $(65.1 \%: \mathrm{p}=0.038)$, whereas no differences in the survivals were observed in the group of patients with serum levels of sCD44s, sCD44v5 either above or below the cut-off value.

From the multivariate analysis, including the known prognostic factors, lymph node metastasis (relative risk $7.351: \mathrm{p}=$ 0.003 ) and the serum levels of sCD44v6 (relative risk 3.256: $\mathrm{p}=0.017$ ), were proven to be independent prognostic factors (Table 4). 
6 Cancer Research and Treatment 2003;35(1)
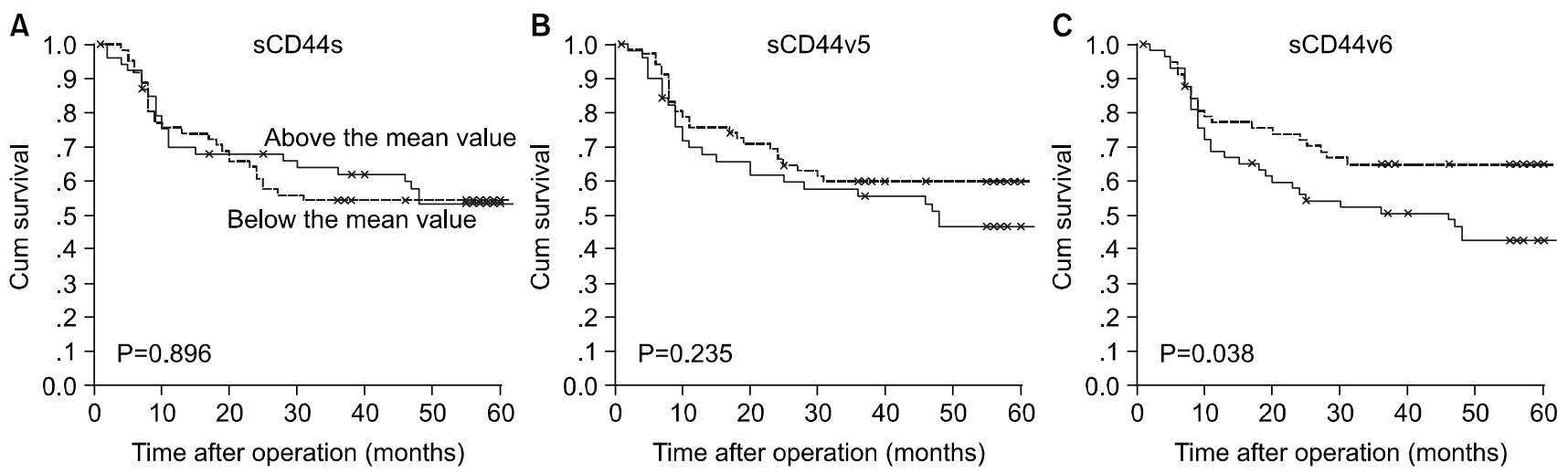

Fig. 1. Survival analysis of patients with gastric cancer is shown in relation to sCD44s, sCD44v5, and sCD44v6 serum levels. The cut-off levels were set at the mean serum levels of sCD44s, sCD44v5, and sCD44v6, respectively (broken line: below the mean value; solid line: above the mean value). No differences were observed in sCD44s (A), and sCD44v5 (B), whereas the 5-year survival rate was significantly lower in high level group of sCD44v6 (C).

Table 4. Multivariate cox regression analysis

\begin{tabular}{lccc}
\multicolumn{1}{c}{ Variable } & $\beta$ & Relative risk & P value \\
\hline Age $(\leq 50$ years, $>50$ years) & 0.186 & 1.205 & 0.639 \\
Sex (female, male) & 0.771 & 2.162 & 0.116 \\
Tumor size $(\leq 4 \mathrm{~cm},>4 \mathrm{~cm}$ ) & 0.352 & 1.789 & 0.195 \\
Gross type (superficial or localized, infiltrative or diffuse) & 0.561 & 1.753 & 0.409 \\
Histologic type (differentiated, undifferentiated) & 0.809 & 1.527 & 0.688 \\
Serosal invasion (none, present) & 1.432 & 3.186 & 0.077 \\
Lymph node metastasis (none, present) & 3.111 & 7.351 & 0.003 \\
Lymphatic invasion (none, present) & 0.809 & 2.247 & 0.178 \\
Venous invasion (none, present) & 0.586 & 0.556 & 0.273 \\
sCD44s level (above mean, below mean) & 0.816 & 0.442 & 0.524 \\
sCD44v5 level (above mean, below mean) & 0.356 & 1.427 & 0.417 \\
sCD44v6 level (above mean, below mean) & 2.181 & 3.256 & 0.017 \\
\hline
\end{tabular}

\section{DISCUSSION}

The soluble CD44s, and its variant isoforms, have been demonstrated in the serum of cancer patients and healthy individuals. Guo et al. (14) first reported increases in the sCD44s serum levels in patients with colon and gastric cancer. Harn et al. (15) demonstrated that the sCD44v5 and sCD44v6 serum levels were elevated in patients with gastric cancer. In addition, they postulated that the soluble CD44, present in the circulation of patients, most likely came from tumor rather than normal cells. At present, however, the exact in vivo function and the cellular source of these soluble CD44 molecules remain uncertain. Because CD44 isoforms are expressed in a wide variety of tissues (18), the question is raised as to whether these proteins, shed from the tumor cells, could serve as indicators of tumor burden and metastasis from their detection in the serum.

In the present study, all the sCD44 proteins, the standard form, as well as the variants, CD44v5 and CD44v6, were found to be present in the serum of healthy individuals. We found also that the serum levels of sCD44s were not significantly different between gastric cancer patients and the healthy controls, and the sCD44v5 was even slightly lower in the gastric cancer patients. Only the preoperative serum levels of sCD44v6 were significantly elevated compared with the healthy controls. A high sCD44v6 serum level was also associated with the venous or lymphatic invasion of the tumor, and the presence of lymph mode metastasis, both being indicative of an invasive phenotype of the gastric cancer. Furthermore, the prognosis of patients with high serum sCD44v6 levels $(>243 \mathrm{ng} / \mathrm{ml})$ was significantly worse than that of patients with low serum levels, and this prognostic significance was maintained by a multivariate analysis on the known prognostic parameters. Although the cutoff level for the best predictive value needs to be clarified with a study of a larger sample of patients, our results suggest that SCD44v6 may be a useful serum marker of tumor invasiveness, and prognosis in patients with gastric cancer. Inversely, Gansauge et al. (19) reported that the serum sCD44v6 
level was significantly reduced in patients with a pancreatic carcinoma, and that a low serum level was significantly associated with a poor prognosis. No significant correlation has been reported between the concentration of the sCD44 isoforms and tumor progression, or prognosis, of ovarian carcinomas (20), renal carcinomas (21) or bladder carcinomas (22). Those results suggest that the role of CD44 in tumor metastasis may be dependent on the species, type of carcinoma or the microenvironment in the host.

Recent studies appear to show that CD44v6 plays an important role in the invasion and metastasis of the diffuse type of gastric cancer, but not of the intestinal type $(10,23)$. However, we have been unable to confirm these findings with this study. We still do not know the reasons for these discrepancies, and further studies are needed to clarify the relationship between the serum sCD44v6 level and the Lauren type of gastric cancer.

The results comparing the pre- and postoperative serum sCD44 levels were very interesting in the present study. When measured on the $7^{\text {th }}$ postoperative day, all the sCD44 proteins were significantly reduced compared to those obtained preoperatively, irrespective of a tumor resection. Moreover, the surgical resection of the tumor masses from these patients resulted in significant reductions in their serum sCD44s, sCD44v5 and sCD44v6 levels. However, with no surgical resection of the tumor there was a significant reduction in the serum sCD44s level only, suggesting that changes in the systemic levels of the $\mathrm{sCD} 44 \mathrm{~s}$ are due, at least in part, to the surgery-induced alterations to the host's immune defense. Sliutz et al. (20) postulated that the production of serum sCD44s in hematopoietic cells overrules the production in ovarian tumor cells, as the serum sCD44s level was higher with high hematopoietic activity. Additional support for this concept was sought by Katoh et al. (24), who observed that the serum levels of sCD44 were significantly reduced in immunodeficient mice, but significantly increased in murine models of autoimmune disease. Guo et al. (25) observed that human immunodeficiency virus infection induced the loss of CD44 in a monocytic cell line. Thus, it is likely that the decrease in the serum sCD44s level is the expression of a reduced number of activated lymphocytes, which could, in turn, be an indication of a depressed immunity in our patients during the postoperative period. Conversely, our data suggested that the isoforms containing sCD44v5 or sCD44v6, detected in the sera of gastric cancer patients, are the result of the tumor cells shedding those isoforms. However, further studies are necessary to elucidate the relation between the serum sCD44v5 or sCD44v6 levels, and the function of the peripheral blood lymphocytes, in patients with gastric cancer.

\section{CONCLUSIONS}

The preoperative serum level of sCD44v6 in patients with gastric cancer is significantly higher than that in healthy controls. The serum sCD44v6 levels correlates with the venous or lymphatic invasion of the tumor and lymph node metastasis. In addition, a high preoperative serum sCD44v6 level was significantly associated with poor prognosis in patients with gastric cancer. These results suggest that the elevation of the serum
sCD44v6 level might be used as a new predictor of tumor invasiveness, and poor prognosis, in patients with gastric cancer.

\section{ACKNOWLEDGEMENTS}

This research was supported, in part, by the Korean Foundation for Cancer Research, Grant No. 99-03.

\section{REFERENCES}

1. Ponta H, Sleeman J, Herrlich P. Tumor metastasis formation: Cell surface proteins confer metastasis-promoting or suppressing properties. Biochem Biophys Acta 1994;1198:1-10.

2. Jalkanen SW, Bargatze RF, De Los Toyos J, Butcher EC. Lymphocyte recognition of high endothelium: antibodies to distinct epitopes of an $85-95 \mathrm{kD}$ glycoprotein antigen differentially inhibit lymphocyte binding to lymph node, mucosal or synovial endothelial cells. J Cell Biol 1987;105:983-990.

3. Terpe HJ, Stark H, Prehm P, Gnthert U. CD44 variant isoforms are preferentially expressed in basal epithelia of non-malignant human fetal and adult tissues. Histochemistry 1994;101:79-89.

4. Miyake K, Underhill CB, Lesley J, Kincade PW. Hyaluronate can function as a cell adhesion molecule and CD44 participates in hyaluronate recognition. J Exp Med 1990;172:69-75.

5. Screaton GR, Bell MV, Jackson DG, Cornelis FB, Gerth U, Bell JI. Genomic structure of DNA encoding the lymphocyte homing receptor CD44 reveals at least 12 alternatively spliced exons. Proc Natl Acad Sci USA 1992;89:12160-12164.

6. Mulder JWR, Kruyt PM, Sewnath M, Seldenrijk CA, Weidema WF, Offerhaus GJA, Pals ST. Colorectal cancer prognosis and expression of exon-v6-containing CD44 proteins. Lancet 1994; 344:1470-1472.

7. Heider KH, Dmmrich J, Skroch-Angel P, Mauller-Hermelink HK, Vollmers HP, Herrlich P, Ponta H. Differential expression of CD44 splice variants in intestinal- and diffuse-type gastric carcinomas and normal gastric mucosa. Cancer Res 1993;53: 4197-4203.

8. Mayer B, Jauch KW, Gnthert U, Figdor CG, Schildberg FW, Funke I, Johnson JP. De-novo expression of CD44 and survival in gastric cancer. Lancet 1993;342:1019-1022.

9. Hong RL, Lee WJ, Shun CT, Chu JS, Chen YC. Expression of CD44 and its clinical implication in diffuse-type and intestinal-type gastric adenocarcinomas. Oncology 1995;52:334-339.

10. Müller W, Schneider A, Heider KH, Meier S, Hommel G, Gabbert HE. Expression and prognostic value of the CD44 splicing variants v5 and v6 in gastric cancer. J Pathol 1997; 183:222-227.

11. Kurozumi K, Nishida T, Nakao K, Nakahara M, Tsujimoto M. Expression of CD44 variant 6 and lymphatic invasion: importance to lymph node metastasis in gastric cancer. World J Surg 1998;22:853-857.

12. Yoo CH, Noh SH, Kim HG, Lee HY, Min JS. Prognostic significance of CD44 and $\mathrm{nm} 23$ expression in patients with stage II and III A gastric carcinoma. J Surg Oncol 1999;71: $22-28$.

13. Ryu KH, Kim HC, Baek MJ, Lee MS, Song OP, Kim CJ. The clinical value of CD44 in gastric cancer. J Korean Cancer Assoc 1999:31:921-930.

14. Guo YJ, Liu G, Wang X, Jin D, Wu M, Ma J, Sy MS. Potential use of soluble CD44 in serum as indicator of tumor burden and metastasis in patients with gastric and colon cancer. Cancer Res 1994;54:422-426. 
15. Harn HJ, Ho LI, Shyu RY, Yuan JS, Lin FG, Young TH, Liu CA, Tang HS, Lee WH. Soluble CD44 isoforms in serum as potential markers of metastatic gastric carcinoma. J Clin Gastroenterol 1996;22:107-110.

16. The Korean Gastric Cancer Association. Korean classification of gastric cancer. 1st ed. Seoul: Eui-hak Publishing, 2002.

17. Sobin $\mathrm{LH}$, Wittekind $\mathrm{CH}$, eds. Union Internationale Contre le Cancer TNM Classification of Malignant Tumors. 5th ed. New York: John Wiley \& Sons, 1997.

18. Fox SB, Faweett J, Jackson DG, Collins I, Gatter KC, Harris AL, Gearing A, Simmons DL. Normal human tissues, in addition to some tumors, express multiple different CD44 isoforms. Cancer Res 1994;54:4539-4546.

19. Gansauge F, Gansauge S, Rau B, Scheiblich A, Poch B, Schoenberg MH, Berger HG. Low serum levels of soluble CD44 variant 6 are significantly associated with poor prognosis in patients with pancreatic carcinoma. Cancer 1997;80: 1733-1739.

20. Sliutz G, Tempfer C, Winkler S, Kohlberger P, Reinthaller A,
Kainz CH. Immunohistochemical and serological evaluation of CD44 splice variants in human ovarian cancer. Br J Cancer 1995;72:1494-1497.

21. Kan M, Kanayama H, Naruo S, Tsuji M, Kojima K, Kurokawa Y, Kagawa S. Serological evaluation of soluble CD44 in renal cancer. J Cancer Res 1996;87:1191-1194.

22. Lein M, Jung K, Weiss S, Schnorr D, Loening SA. Soluble CD44 variants in the serum of patients with urological malignancies. Oncology 1997;54:226-230.

23. Saito H, Tsujitani S, Katano K, Ikeguchi M, Maeta M, Kaibara N. Serum concentration of CD44 variant 6 and its relation to prognosis in patients with gastric carcinoma. Cancer 1998;83: 1094-1101.

24. Katoh S, McCarthy JB, Kincade PW. Characterization of soluble CD44 in the circulation of mice: levels are affected by immune activity and tumor growth. J Immunol 1994;153: 3440-3449.

25. Guo MM, Hildreth JE. HIV-induced loss of CD44 expression in monocytic cell lines. J Immunol 1993;151:2225-2236. 